\title{
NUMERICAL ANALYIS OF THE LOW-ALTITUDE AIR TURBULENCE MATHEMATICAL MODELS USED IN MODELLING OF THE SPATIAL MOTION OF THE SMALL UNMANNED AERIAL VEHICLES
}

\author{
Róbert SZABOLCSI \\ Óbuda University, Budapest, Hungary \\ szabolcsi.robert@bgk.uni-obuda.hu
}

\begin{abstract}
Unmanned Aerial Vehicles designed and manufactured often with unknown origin but available in sale of the model aircraft market rarely deals with weather circumstances and conditions defining clearances for safe air operations of the UAV. UAVS used in low-altitude flight missions are often threatened by atmospheric turbulences leading either to high angle-of-attack (AoA) or leading to the stall of the UAV. There are many mathematical models well-known and widely applied in piloted aircraft aviation when to simulate atmospheric turbulences affecting spatial motion of the aircraft. This paper targets to evaluate and simulate numerically the low-altitude air turbulences, and, to examine the vertical gust speed of the small UAV. The UAV behaviour examined numerically will support to find weather clearances ensuring UAV flight safety having the level equal or higher to that level of manned aircraft regulated well-before. A computer code in MATLAB environment is created to support numerical analysis of the small UAV behaviour in low altitude atmospheric turbulence.
\end{abstract}

Keywords: air turbulence, modelling atmospheric turbulences, small UAV in turbulent air, numerical simulation

\section{Introduction}

Small UAVs are in the focus of attention of many researches due to its popularity and broadband applications. The deep impact has been shown by several small UAV applications both in the field of military and non-military applications.

The well-organized UAV flight is reach of weather conditions evaluation however, there are many UAV users are skipping thorough analysis of weather reports leading to several emergency situations, or to a fatal consequence of the crash of the UAV.

The atmospheric turbulence has two main mathematical models widely applied analysis and design of the automatic flight control systems. Deterministic gust models, such as step, or, unit step function, or, '1cos' gust model are used to evaluate weather clearances. Turbulence models can be classified by the altitude to whom itself belonging. This article deals with low altitude atmospheric turbulence models derived for height of the flight less than $2000 \mathrm{ft}$. For small UAVs flight envelope limited by such altitudes will cover almost all possible flight scenario. The purpose of the author is to create virtual environment in the form of the computer code used in simulation of the atmospheric turbulences, namely the speed components of the random gust speed, say, $u_{g}, \mathrm{v}_{g}$, and $w_{g}$ will be simulated, and finally, a numerical example will be presented.

\section{Preliminaries and Literature Review \\ G. Korn's excellent book is a general frame}


deriving main and robust theoretical backgrounds for random signals and random systems [1]. D. McLean is a pioneer in modern interpretation and propagation of the analysis and design of the automatic flight control systems. In his work [2] he demonstrates both deterministic and random mathematical models of the atmospheric turbulences. After many years and decades, knowledges splitted and puzzled into many foregoing military standards (MIL-STD) were compiled and integrated into unique ones, such as demonstrated by [3]. The computer simulation of the atmospheric turbulences, theirs application in analysis and design of the automatic flight control systems outlined in references of $[4,5,6,7,8,9]$.

Stochastic models of the gust speed components are created using computer package MATLAB $^{\circledR}$ [10], and Control System Toolbox [11].

3. Mathematical models of the atmospheric turbulences applied in small UAV flight simulations

Using turbulent air speed components' records, the power spectral density (PSD) function was determined. The more precise form of the PSD function is named after Theodore von Kármán, and can be derived as follows below [2, 3, 4, 5, 6, 7, 8, 9]:

$\Phi_{\text {Kármán }}(\Omega)=\frac{\sigma^{2} L}{\pi} \frac{1+\frac{8}{3}(1,339 L \Omega)^{2}}{\left(1+1,339 L^{2} \Omega^{2}\right)^{11 / 6}}$

In equation (1) $L[\mathrm{~m}]$ is the gust wavelength, $\Omega=\omega U_{0}^{-1}[\mathrm{rad} / \mathrm{m}]$ is the spatial frequency, $\omega[\mathrm{rad} / \mathrm{s}]$ is the angular frequency, and finally, $\sigma[\mathrm{m} / \mathrm{s}]$ is the $R M S$ of the gust velocity.

The second PSD function widely used is the so-called Dryden PSD function, which can be defined as given below $[2,3$, $4,5,6,7,8,9]$ :

$$
\Phi_{\text {Dryden }}(\Omega)=\frac{\sigma^{2} L}{\pi} \frac{1+3 L^{2} \Omega^{2}}{\left(1+L^{2} \Omega^{2}\right)^{2}}
$$

For rigid-body UAV the simplest mathematical form of the PSD function defined by equation of (2) will be used in this paper. References of [1, 2, 3, 4] define PSD functions of the air speed components of the turbulent air along body axis system of the UAV in the following manner:

$$
\begin{gathered}
\Phi_{\mathrm{u}_{\mathrm{g}}}(\Omega)=\frac{2 \sigma_{\mathrm{u}}^{2} L_{\mathrm{u}}}{\pi} \frac{1}{1+\left(L_{\mathrm{u}} \Omega\right)^{2}} \\
\Phi_{\mathrm{v}_{\mathrm{g}}}(\Omega)=\frac{\sigma_{\mathrm{v}}^{2} L_{\mathrm{v}}}{\pi} \frac{\left(1+3\left(L_{\mathrm{v}} \Omega\right)^{2}\right)}{\left[1+\left(L_{\mathrm{v}} \Omega\right)^{2}\right]^{2}} \\
\Phi_{\mathrm{w}_{\mathrm{g}}}(\Omega)=\frac{\sigma_{\mathrm{w}}^{2} L_{\mathrm{w}}}{\pi} \frac{\left(1+3\left(L_{\mathrm{w}} \Omega\right)^{2}\right)}{\left[1+\left(L_{\mathrm{w}} \Omega\right)^{2}\right]^{2}}
\end{gathered}
$$

where $\sigma_{i}^{2}=\left.\int_{0}^{\infty} \Phi_{i}(\Omega) d \Omega_{i}\right|_{i=u, v, \text { or } w}$.

It is well-known that, $\omega=U_{o} \Omega$, equations of (3)-(5) may be rewritten as:

$$
\begin{gathered}
\Phi_{\mathrm{u}_{\mathrm{g}}}(\omega)=\frac{2 \sigma_{\mathrm{u}}^{2} L_{\mathrm{u}}}{U_{o} \pi} \frac{1}{\left\{1+\left(L_{\mathrm{u}} / U_{o}\right)^{2} \omega^{2}\right\}} \\
\Phi_{\mathrm{v}_{\mathrm{g}}}(\omega)=\frac{\sigma_{\mathrm{v}}^{2} L_{\mathrm{v}}}{U_{o} \pi} \frac{\left(1+3\left(L_{\mathrm{v}} / U_{o}\right)^{2} \omega^{2}\right)}{\left\{\left(1+\left(L_{\mathrm{v}} / U_{o}\right)^{2} \omega^{2}\right\}^{2}\right.} \\
\Phi_{\mathrm{w}_{\mathrm{g}}}(\omega)=\frac{\sigma_{\mathrm{w}}^{2} L_{\mathrm{w}}}{U_{o} \pi} \frac{\left(1+3\left(L_{\mathrm{w}} / U_{o}\right)^{2} \omega^{2}\right)}{\left\{\left(1+\left(L_{\mathrm{w}} / U_{o}\right)^{2} \omega^{2}\right\}^{2}\right.}
\end{gathered}
$$

Random signals can be generated and filtered from wide-band noise generator having PSD function of $\Phi_{N}(\omega)$ and, a linear filter must be introduced to ensure output signal PSD function of $\Phi_{i}(\omega)$ (Figure 1.) $[2,4,8,9]$ :

$$
\begin{aligned}
& \Phi_{i}(\omega)=\left|G_{i}(s)\right|_{s=j \omega}^{2} \Phi_{N}(\omega)= \\
& =\left.G_{i}(s) G_{i}(-s)\right|_{s=j \omega} \Phi_{N}(\omega)
\end{aligned}
$$

If a wide-band signal generator has the unit intensity over an infinite frequency range, the signal generator is called as 'white noise' source having PSD function as given below:

$\Phi_{N}(\omega)=1$ 


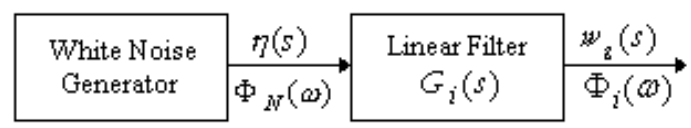

Figure 1: Block Diagram of Filtering Stochastic Signals.

Substitution of eq (10) into eq (9) results in the following formula:

$$
\begin{aligned}
& \Phi_{i}(\omega)=\left|G_{i}(s)\right|_{s=j \omega}^{2} \Phi_{N}(\omega)= \\
& =\left.G_{i}(s) G_{i}(-s)\right|_{s=j \omega}
\end{aligned}
$$

The linear filter transfer functions of $G_{i}(s)$ providing filter outputs equal to that of famous speed components of the turbulent air are given in $[2,9]$ to be:

$$
\begin{aligned}
& G_{\mathrm{u}_{\mathrm{g}}}(s)=\frac{\sqrt{K_{\mathrm{u}}}}{s+\lambda_{\mathrm{u}}} \\
& G_{\mathrm{v}_{\mathrm{g}}}(s)=\sqrt{K_{\mathrm{v}}} \frac{\mathrm{s}+\beta_{\mathrm{v}}}{\left(s+\lambda_{\mathrm{v}}\right)^{2}} \\
& G_{\mathrm{w}_{\mathrm{g}}}(s)=\sqrt{K_{\mathrm{w}}} \frac{\mathrm{s}+\beta_{\mathrm{w}}}{\left(s+\lambda_{\mathrm{w}}\right)^{2}}
\end{aligned}
$$

Constants of equations of (12-1)-(123) are as listed below:

$$
\begin{aligned}
& K_{\mathrm{u}}=\frac{2 U_{o} \sigma_{\mathrm{u}}^{2}}{L_{\mathrm{u}} \pi}, K_{\mathrm{v}}=\frac{3 U_{o} \sigma_{\mathrm{v}}^{2}}{L_{\mathrm{v}} \pi}, K_{\mathrm{w}}=\frac{3 U_{o} \sigma_{\mathrm{w}}^{2}}{L_{\mathrm{w}} \pi} \\
& \beta_{\mathrm{v}}=\frac{U_{o}}{\sqrt{3} L_{\mathrm{v}}}, \beta_{\mathrm{w}}=\frac{U_{o}}{\sqrt{3} L_{\mathrm{w}}} \\
& \lambda_{\mathrm{u}}=\frac{U_{o}}{L_{\mathrm{u}}}, \lambda_{\mathrm{v}}=\frac{U_{o}}{L_{\mathrm{v}}}, \lambda_{\mathrm{w}}=\frac{U_{o}}{L_{\mathrm{w}}}
\end{aligned}
$$

This paper addresses low altitude low speed air turbulence models let us define initial flight parameters of the small UAV being investigated to be as follows below:

$$
\begin{gathered}
H=100 \mathrm{~m} \cong 328,084 \mathrm{feet}, \\
U_{0}=25 \mathrm{~m} / \mathrm{s}=90 \mathrm{~km} / \mathrm{h}
\end{gathered}
$$

It is easy to see that parameters of the transfer functions of eq (12) are necessary to know to parametrize mathematical model of the linear filters, in other words, it is necessary to know turbulence scale of $L_{i}$, and turbulence intensity of $\sigma_{i}$.
References [2, 3, 8, 9] tabulate air turbulence RMS parameters used by NASA as follows below:

$$
\begin{aligned}
& 3,4 \mathrm{~m} / \mathrm{s} \leq \sigma_{u} \leq 0,85 \mathrm{~m} / \mathrm{s} \\
& 2,8 \mathrm{~m} / \mathrm{s} \leq \sigma_{v} \leq 0,7 \mathrm{~m} / \mathrm{s} \\
& 1,8 \mathrm{~m} / \mathrm{s} \leq \sigma_{w} \leq 0,45 \mathrm{~m} / \mathrm{s}
\end{aligned}
$$

Although normal weather conditions are investigated, extreme weather conditions (thunderstorm) also are in the focus of attention. References [2, 3] suggest turbulence intensities to be as follows:

$$
\sigma_{u}=\sigma_{v}=\sigma_{w}=7 \mathrm{~m} / \mathrm{s}
$$

The integral scale lengths $L_{i}$ of the low altitude atmospheric turbulences in between of the height of the flight 10 feet $\leq h \leq 1000$ feet can be derived using equations given to be $[2,3,5,8,9]$ :

$$
\begin{aligned}
& L_{u}=2 L_{v}=\frac{h}{(0,177+0,000823 \cdot h)^{1,2}} \\
& L_{w}=0,5 h
\end{aligned}
$$

For extreme weather conditions one can apply following integral scale lengths $[2,3,8,9]$ :

$$
L_{u}=L_{v}=L_{w}=580 \mathrm{~m}
$$

For the low altitude random turbulence models intensity of the turbulence, $\sigma_{w}$ can be measured as:

$$
\sigma_{w}=0,1 u_{20}
$$

where $u_{20}$ is constant longitudinal component speed of the turbulent air measured at the altitude of $h=20$ feet. Using equations of (21)-(22) integral scale lengths of the air turbulence were found and they are summarized in Table 1. 
Table 1 Integral Scale Lengths at $H=100 \mathrm{~m}$

\begin{tabular}{c|c}
\hline Turbulence Integral Scale Length $(L)[\mathrm{m}]$ & Nominal \\
\hline$L_{\mathrm{u}}$ & $862,185497 \mathrm{feet} \cong 262,7941311 \mathrm{~m}$ \\
$L_{\mathrm{v}}=0,5 L_{u}$ & $431,0927485 \mathrm{feet} \cong 131,3970655 \mathrm{~m}$ \\
$L_{\mathrm{w}}$ & $50 \mathrm{~m}$ \\
\hline
\end{tabular}

Using equations of (17)-(20) turbulence tabulated in Table 2. intensities were found and they are

Table 2 Turbulence Intensities

\begin{tabular}{c|c}
\hline Turbulence Intensities, $[\mathrm{m} / \mathrm{s}]$ & NASA-Min \\
\hline$\sigma_{\mathrm{u}},[\mathrm{m} / \mathrm{s}]$ & 0,85 \\
$\sigma_{\mathrm{v}},[\mathrm{m} / \mathrm{s}]$ & 0,7 \\
$\sigma_{\mathrm{w}},[\mathrm{m} / \mathrm{s}]$ & 0,45 \\
\hline
\end{tabular}

Constant speed of the turbulent air, denoted as $\mathrm{u}_{20}$, was found using military standard of [3], and using equations of (21)-(22).
Constant speed parameters of $\mathrm{u}_{20}$ are summarized in Table 3.

Table 3 UAV Constant Speed, $\mathrm{u}_{20}$

\begin{tabular}{c|c}
\hline Turbulent Air Characteristics & NASA-Min \\
\hline$\sigma_{\mathrm{w}}=0,1 u_{20},[\mathrm{~m} / \mathrm{s}]$ & 0,45 \\
$\mathrm{u}_{20},[\mathrm{~m} / \mathrm{s}]-[\mathrm{km} / \mathrm{h}]$ & 4,5 \\
\hline
\end{tabular}

Linear transfer functions defined by equations (12) having parameters given by equations of (13)-(15), and satisfying conditions derived by equations (16)-(24), and considering weather conditions given by Table 1, and Table 2, can be determined, and they are tabulated in Table 4, Table 5, and Table 6, respectively $[2,3,8,9]$ :

Table 4 Parameters of the linear filters providing speed components of the air turbulence, $\mathrm{u}_{\mathrm{g}}(t)$

\begin{tabular}{c|c}
\hline Turbulent Air Characteristics for NASA-Min \\
\hline$K_{\mathrm{u}}=\frac{2 \sigma_{\mathrm{u}}^{2} U_{o}}{L_{\mathrm{u}} \pi}\left[\mathrm{m}^{2} / \mathrm{s}^{3}\right]=0,04375$ & $\lambda_{\mathrm{u}}=\frac{U_{o}}{L_{\mathrm{u}}}\left[\mathrm{s}^{-1}\right]=0,09513$ \\
\hline
\end{tabular}

Table 5 Parameters of the linear filters providing lateral speed component of the air turbulence,

$$
\mathrm{v}_{\mathrm{g}}(t) \text {. }
$$




\begin{tabular}{c|l|l}
\hline \multicolumn{3}{c}{ Turbulent Air Characteristics for NASA-Min } \\
\hline$K_{\mathrm{v}}=\frac{3 \sigma_{\mathrm{v}}^{2} U_{o}}{L_{\mathrm{v}} \pi}\left[m^{2} / \mathrm{s}^{3}\right]=0,08902$ & $\beta_{\mathrm{v}}=\frac{U_{o}}{\sqrt{3} L_{\mathrm{v}}}\left[s^{-1}\right]=0,10984$ & $\lambda_{\mathrm{v}}=\frac{U_{o}}{L_{\mathrm{v}}}\left[s^{-1}\right]=0,19026$ \\
\hline
\end{tabular}

Table 6 Parameters of the linear filters providing vertical speed component of the air turbulence, $\mathrm{w}_{\mathrm{g}}(t)$.

\begin{tabular}{c|c|c} 
Turbulent Air Characteristics for NASA-Min & $\mathrm{w}_{\mathrm{g}}(t)$. \\
\hline$K_{\mathrm{w}}=\frac{3 \sigma_{\mathrm{w}}^{2} U_{o}}{L_{\mathrm{w}} \pi}\left[\mathrm{m}^{2} / \mathrm{s}^{3}\right]=0,09668$ & $\beta_{\mathrm{w}}=\frac{U_{o}}{\sqrt{3} L_{\mathrm{w}}}\left[\mathrm{s}^{-1}\right]=0,28867$ & $\lambda_{\mathrm{w}}=\frac{U_{o}}{L_{\mathrm{w}}}\left[s^{-1}\right]=0,5$ \\
\hline
\end{tabular}

Using data tabulated in Table 4, Table 5, and Table 6, transfer functions used to filter appropriate speed components of the turbulent air can be found as shown below:

$$
\begin{aligned}
& G_{\mathrm{u}_{\mathrm{g}}}^{M i n}(s)=\frac{0,20918}{s+0,09513} \\
& G_{\mathrm{v}_{\mathrm{g}}}^{M i n}(s)= \\
& =0,29837 \frac{\mathrm{s}+0,10984}{s^{2}+0,38052 s+0,03620} \\
& G_{\mathrm{w}_{\mathrm{g}}}^{M i n}(s)=0,31094 \frac{\mathrm{s}+0,28867}{s^{2}+s+0,25}
\end{aligned}
$$

4. Results of the computer numerical simulation

Using filtering system introduced in Figure 1 , and using transfer functions of the linear filters defined by equations of (24)-(26) a computer code using MATLAB ${ }^{\circledR} 9.0$ [10] supplemented with Control System Toolbox 10.0 [11] was elaborated by the author.

Figure 2 demonstrates time series as input signal of the linear filter shown in Figure 1 $[10,11]$.

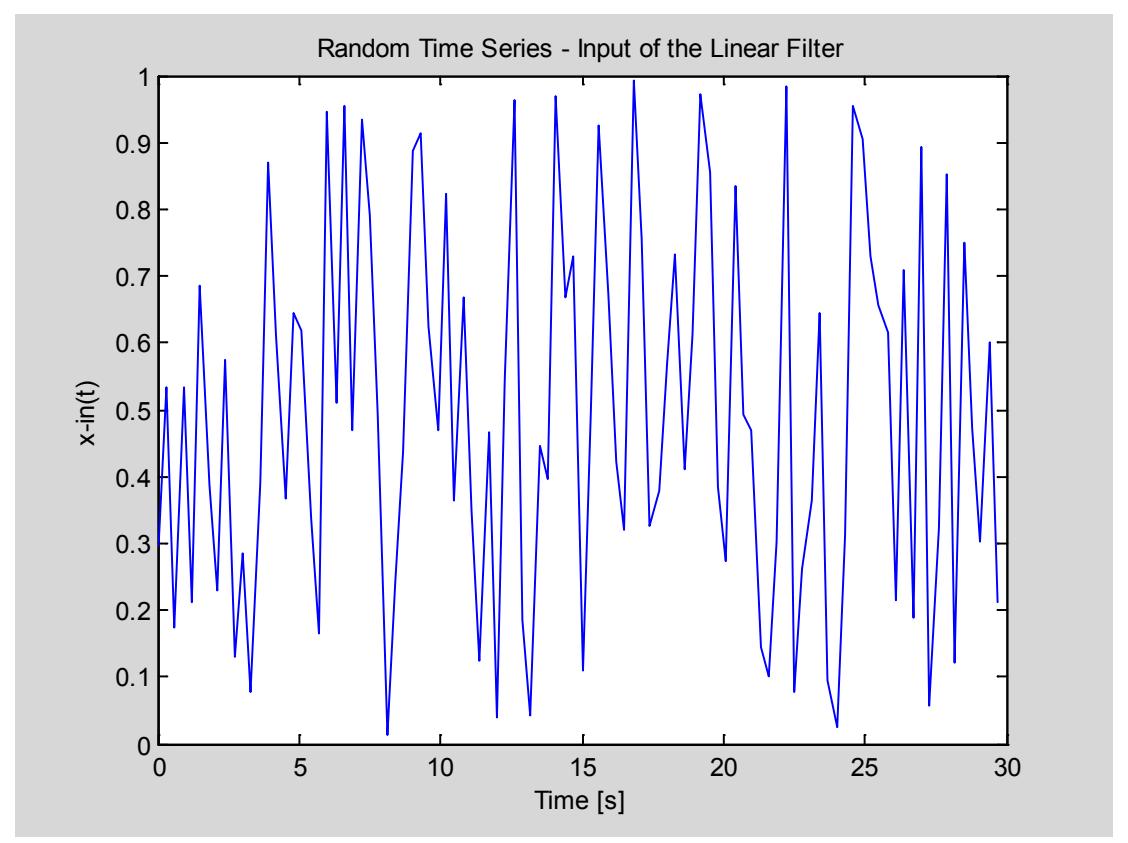

Figure 2: Random time series behaviour.

Using idea of filtering out time series of the speed component of the air turbulence along axes of the UAV body-axes coordinate system speed components were 
calculated using filter transfer functions of (24)-(26) $[7,8,9]$. Results of the computer

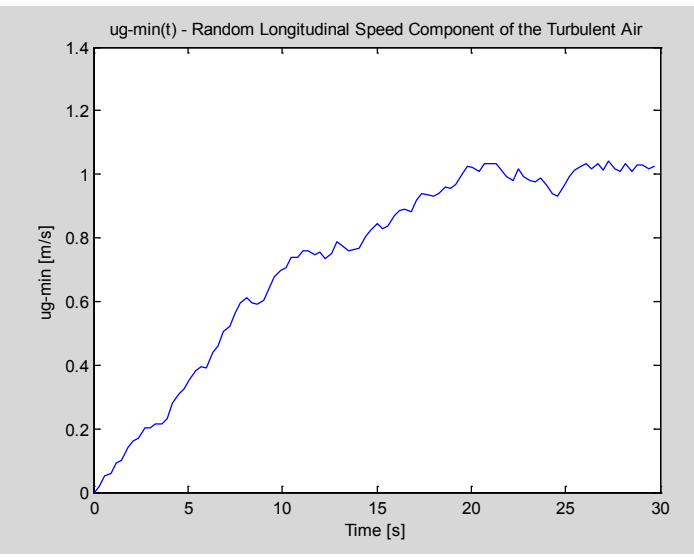

a) simulation can be seen in Figure $3[10,11]$.

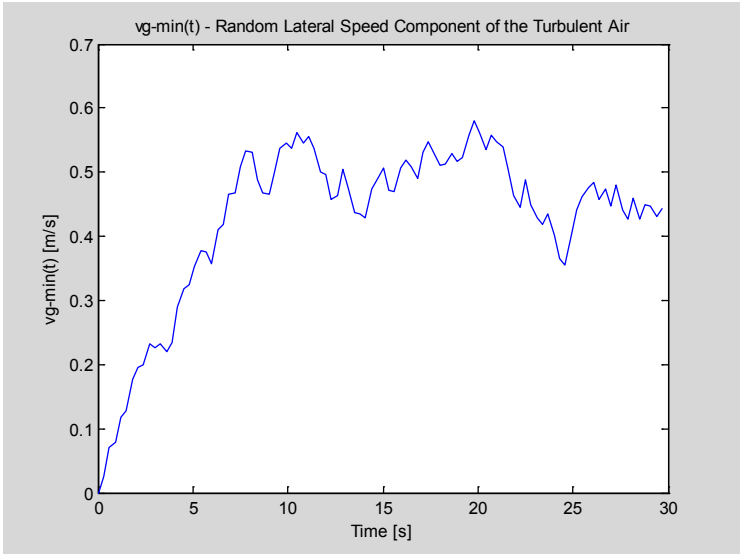

b)

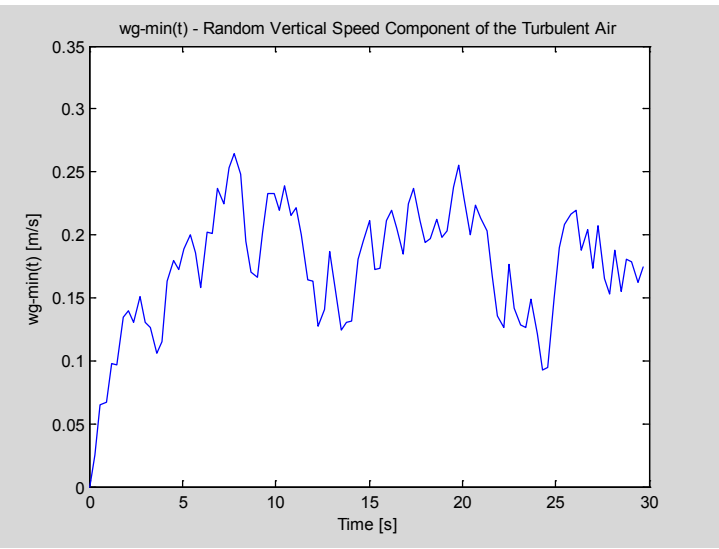

c)

Figure 3: Random speed components.

From Figure 3 it is easy to determine that in the first 10 , or 15 seconds of the transient time result must be eliminated due to physics of the atmosphere [2, 3].
Figure 4 demonstrates behavior of the longitudinal speed component of $\mathrm{u}_{\mathrm{g}}(t)$, simulated using data tabulated in Table 4.

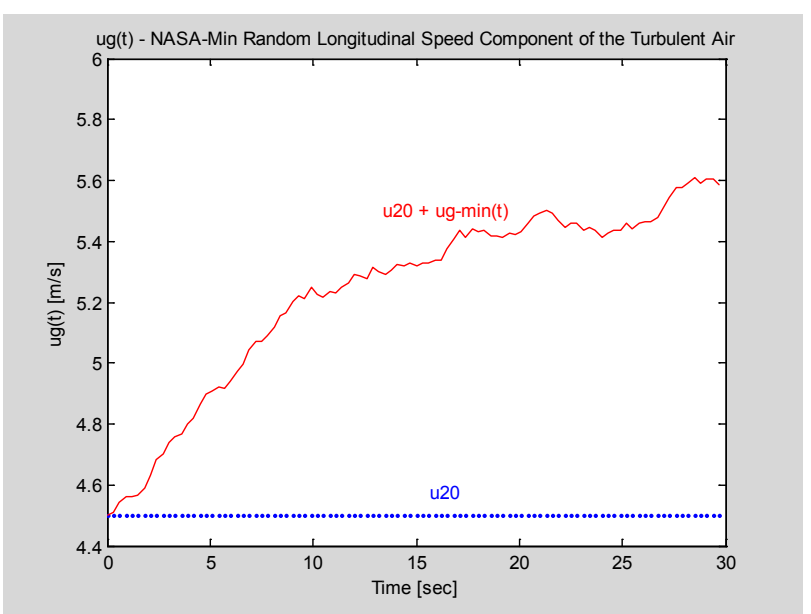

Figure 4: Deterministic constant and stochastic longitudinal speed components.

The three speed components of the atmospheric turbulence can be seen in 
Figure $5[10,11]$. From Figure 5 it can be component has the smallest value. easily derived that vertical gust speed

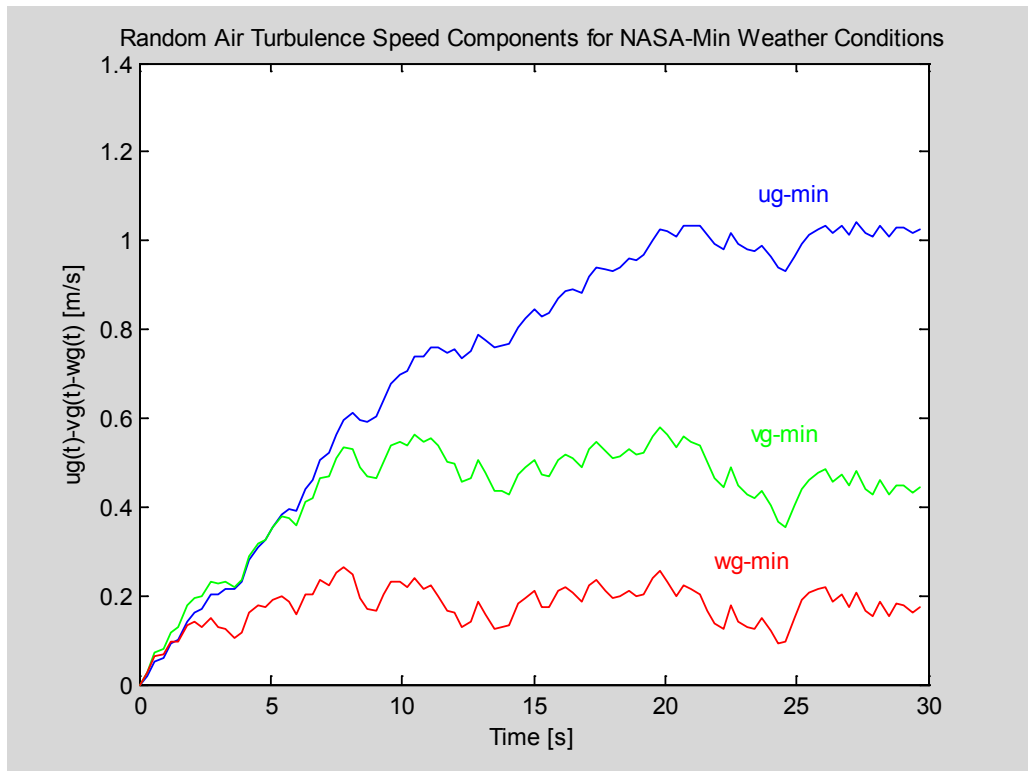

Figure 5: Speed components of the turbulent air.

\section{A numerical example}

An example explained below is based on flight dynamics data of the Boomerang-60 'Trainer' small UAV. The UAV state space model of the short period longitudinal motion at low altitudes and at speed of $U_{o}=19 \mathrm{~m} / \mathrm{s}=68,4 \mathrm{~km} / \mathrm{h}$ is as follows [9]:

$$
\begin{aligned}
& \dot{\mathbf{x}}=\mathbf{A} \mathbf{x}_{s p}+\mathbf{B} \mathbf{u}_{s p}=\left[\begin{array}{cc}
-0,9966 & 19 \\
-3,9794 & -12,991
\end{array}\right]\left[\begin{array}{l}
w \\
q
\end{array}\right]+ \\
& +\left[\begin{array}{c}
-1,2965 \\
-18,7890
\end{array}\right]\left[\delta_{e}\right]
\end{aligned}
$$

where $w$ is the vertical speed of the UAV, $q$ is the pitch rate of the UAV, and, finally, $\delta_{e}$ is the elevator deflection. The UAV model given by equation (27) is a single input-multi output multivariable one.

This model is reduced to that of the single input-single output one, when to take into consideration only vertical speed of $w$ as the UAV response. Omitting pitch rate as UAV response the dynamical model can be re- written as given below:

$$
\dot{w}=-0,9966 w-1,2965 \delta_{e}
$$

If to produce nose-down deflection of the UAV elevator so as to get vertical speed change being positive one, and re-arranging eq (28) yields to the following formula: $\dot{w}+0,9966 w=1,2965 \delta_{e}$

Taking Laplace-transform of eq (29) regarding zero initial conditions results in:

$$
Y(s)=\frac{w(s)}{\delta_{e}(s)}=\frac{1,2965}{1+1,0034 s}
$$

The 'Trainer' UAV was tested for the unit step change of the elevator deflection, say $\delta_{e}(t)=1(t)$. Results of the computer simulation can be seen in Figure 6. [10, 11]. From Figure 6 it can be seen that UAV behavior is the exponential one with the steady-state value of the vertical speed of $1,2965 \mathrm{~m} / \mathrm{s}$. 


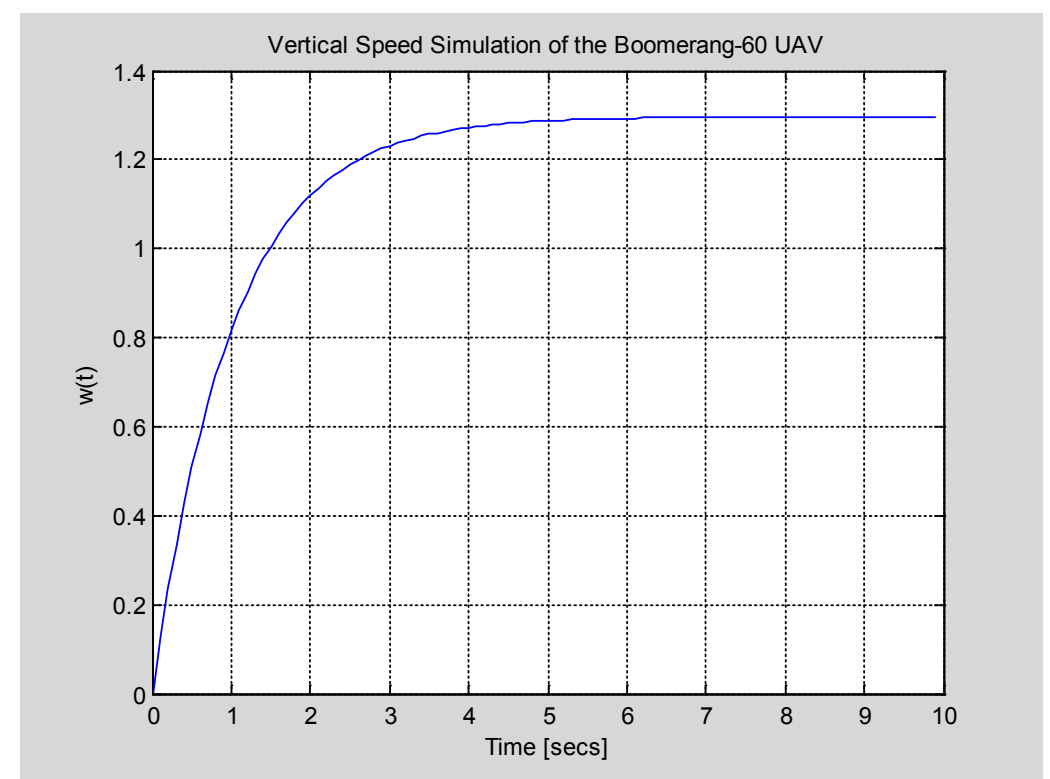

Figure 6: Unit step response of the Trainer UAV.

It is obvious, that UAV resulting vertical speed in turbulent air can be derived as:

$$
w_{\text {res }}=w_{\text {uav }}+w_{\text {wind }}
$$

i.e. resulting vertical speed is a sum of two speed components, i.e. first term in eq (31) derives the vertical speed component of the
UAV, the second term represents the vertical speed component of the atmospheric turbulence of small winds. Results of the computer numerical simulation can be seen in Figure $7[10,11]$.

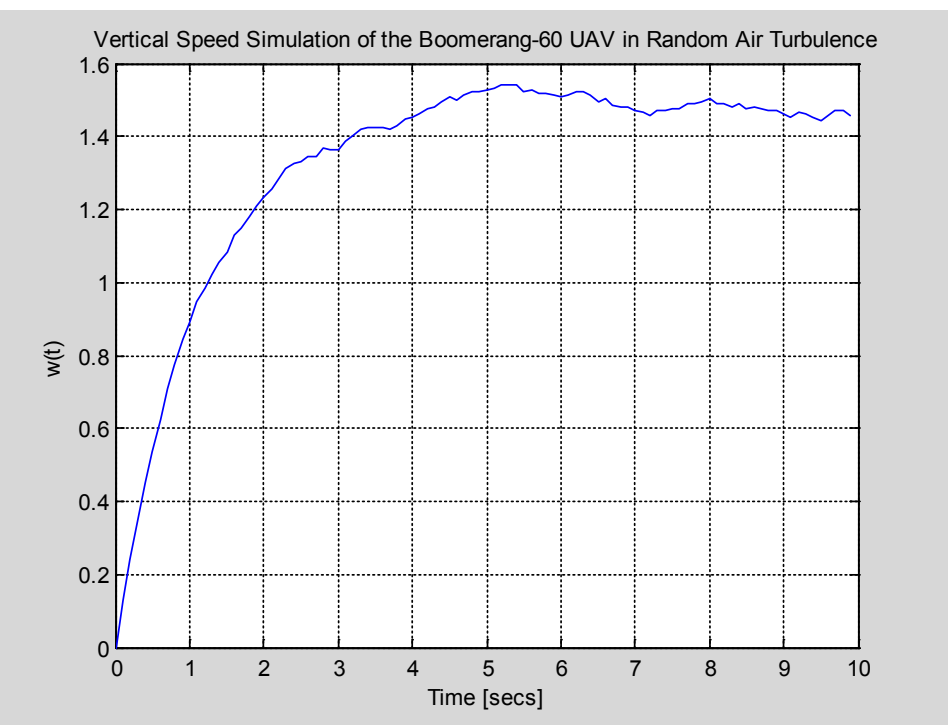

Figure 7: Unit step response of the Trainer UAV in atmospheric turbulence.

Regarding flight safety criteria the UAV flight is often limited by its angle-of-attack, often denoted as $\alpha$ (AoA). So as to avoid critical value of the AoA and to prevent UAV of stalling, it is necessary to know AoA and in case of necessity AoA must be limited to that of safe values less than critical one. For small angles it is evident that $[2,9]$ :

$w \cong U_{o} \alpha$

where $U_{o}$ is the equilibrium speed of the UAV. Results of the computer numerical simulation of the UAV AoA can be seen in Figure $8[10,11]$. 


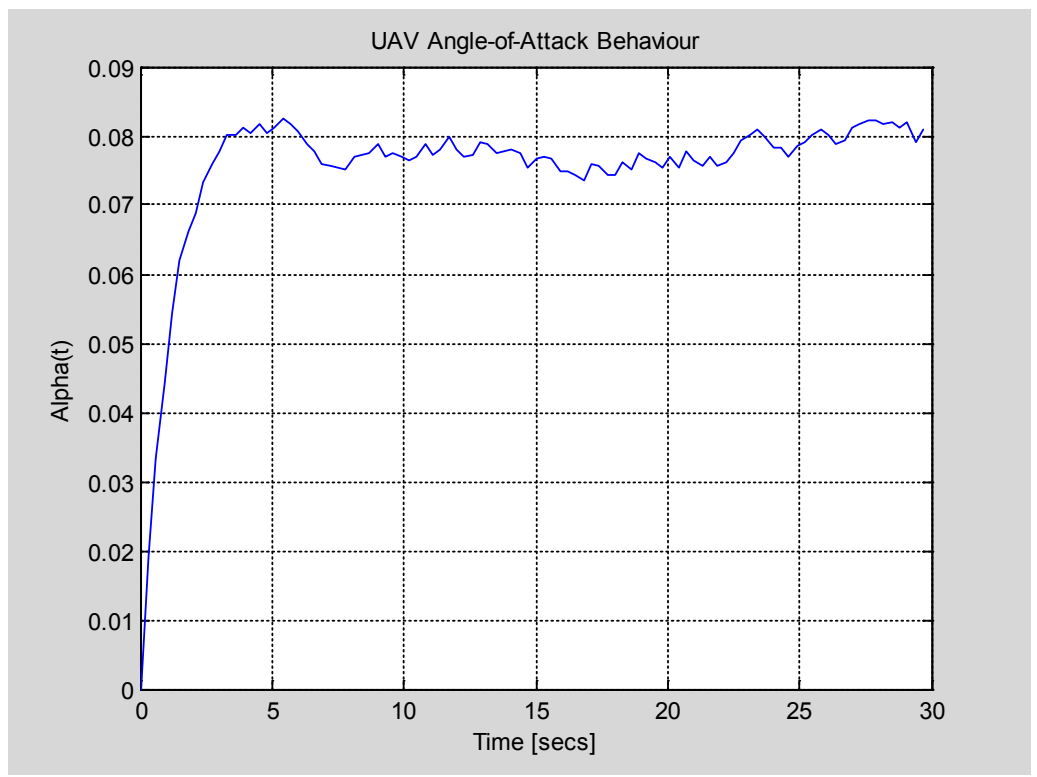

Figure 8: UAV AoA behaviour.

From Figure 8 it is evident that AoA has random feature, however it could not lead to critical angle-of-attack value, i.e. flight safety is ensured with equilibrium initial value of the angle-of-attack.

In low altitude UAV missions it is important to know how the UAV altitude of $h(\mathrm{t})$ will alter from the equilibrium height of the flight ensuring safe UAV flight. UAV altitude can be found if to integrate resulting vertical speed of $w_{\text {res }}$, i.e.:

$h(t)=\int w_{\text {res }} d t$

UAV altitude behavior was simulated and it can be evaluated using Figure $9[10,11]$.

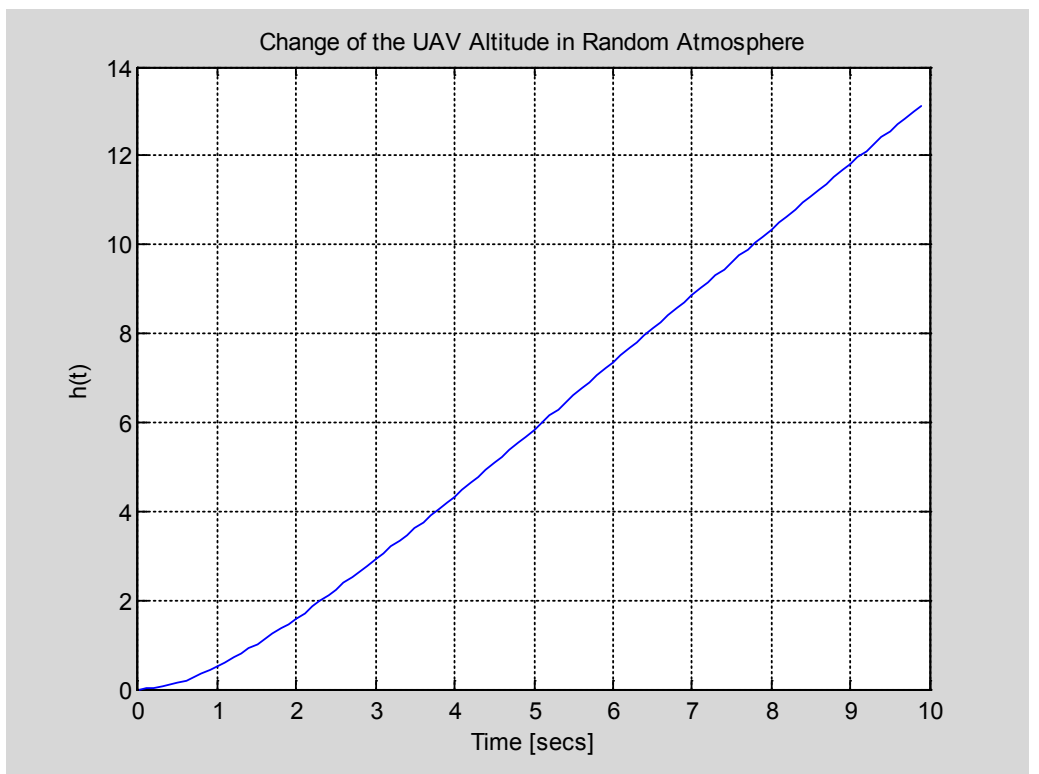

Figure 9: UAV altitude time domain behaviour.

From Figure 9 it is easily can be seen that there is a meaningful change in height of the UAV flight, i.e. the first five seconds of the transient results in change of UAV altitude of 6 meters, which can be a large one relative to the small height of the flight of the UAV measured from the surface of any kind. 


\section{Discussion and conclusions}

Among those of all possible UAV low altitude flight regimes the most dangerous ones are take-off and landing of the UAV. Small UAVs at these low altitude flight phases are mostly controlled manually, and, if there is no on-board autopilot to eliminate weaknesses of the operator, the UAV can be crashed.

Difficult flight situations are mainly caused by atmospheric turbulences. Behaviour of the low altitude atmospheric turbulences was investigated using numerical computer simulation using MATLAB computer package supplemented with Control System Toolbox.

First important finding is that response of the UAV in the control channel of the 'Elevator' is mainly determined by angular deflection of the elevator, and, additional term in vertical speed of the UAV caused by vertical speed component of the turbulent air is a negligible small one.
The AoA change due to vertical wind speed was also analysed. It can be stated that 'light wind' weather conditions would not result in meaningful change of the angle-ofattack, AoA, i.e. probability to reach critical angles, or, as fatal, to stall the UAV is very low.

Regarding UAV behaviour in longitudinal motion, UAV altitude will change very intensively, i.e. UAV is able to manoeuvre very rapidly, although elevator is deflected for the unit angle. If there is a need to conduct manoeuvres more rapidly, the greater elevator deflection must be introduced.

This paper deals with weather clearances of the light winds. If there is a need of evaluation of the UAV behaviour in more unfriendly environment, the wind speed components must be generated for the given weather conditions. For that activity references $[2,3,4,6,7,8,9]$ can be used very effectively.

\section{References}

[1] Granino Korn Random-Process Simulation and Measurements. McGraw-Hill Book Company, New York-Toronto-London-Sydney, 1966.

[2] Donald McLean Automatic Flight Control Systems. Prentice-Hall, Int., New York London - Toronto - Sydney - Tokyo - Singapore, 1990.

[3] MIL-STD-1797A, Notice 3, Flying Qualities of Piloted Aircraft, Department of Defense, Interface Standard, 2004.

[4] Róbert Szabolcsi Mathematical Models for Gust Modeling Applied in Automatic Flight Control Systems' Design, Proceedings of the " 5 th International Conference New Challenges in the Field of Military Sciences 2007”, Vol. II., ISBN 978-963-87706-0-8, pp (95-118), 2007, Budapest, Hungary.

[5] Róbert Szabolcsi Preliminary Control Law Synthesis for Automatic Flight control Systems of Aircraft, Proceedings of the " 5 th International Conference New Challenges in the Field of Military Sciences 2007”, Vol. II., ISBN 978-963-87706-0-8, pp (119-136), 2007, Budapest, Hungary.

[6] Róbert Szabolcsi - György Mészáros Computer Aided Simulation of the Random Atmospheric Turbulences, CD-ROM Proceedings of the $6^{\text {th }}$ International Conference on Crisis Management, ISBN 978-80-7231-510-9, pp(366-379), 14-15 May, 2008, Brno, Czech Republic

[7] Róbert Szabolcsi Stochastic Noises Affecting Dynamic performances of the Automatic Flight Control Systems, Review of the Air Force Academy, ISSN 1842-9238, 1/2009., $\mathrm{pp}(23-30)$.

[8] Róbert Szabolcsi Modern Automatic Flight Control Systems, Miklós Zrínyi National Defense University, Budapest, ISBN 978-963-7060-32-8, p415, 2011 (in Hungarian). 
[9] Róbert Szabolcsi Automatic Flight Control Systems of the Air Robots, Óbuda University, Budapest, ISBN 978-615-5460-23-4, p478, 2016 (in Hungarian).

[10] MATLAB ${ }^{\circledR} 9.0$ (R2016a) - The Language of Technical Computing, User's Guide, The MathWorks, Inc., 2016.

[11] Control System Toolbox 10.0 for Use with MATLAB ${ }^{\circledR}$ (R2016a), User's Guide, The MathWorks, Inc., 2016. 